Meta

Journal des traducteurs

Translators' Journal

\title{
Les mots du transport et de la gestion : approche sémantique-contextuelle
}

\section{Mercedes Eurrutia Cavero}

Volume 50, numéro 4, décembre 2005

Pour une traductologie proactive - Actes

For a Proactive Translatology - Proceedings

Por una traductología proactiva - Actas

URI : https://id.erudit.org/iderudit/019915ar

DOI : https://doi.org/10.7202/019915ar

Aller au sommaire du numéro

Éditeur(s)

Les Presses de l'Université de Montréal

ISSN

0026-0452 (imprimé)

1492-1421 (numérique)

Découvrir la revue

Citer cet article

Eurrutia Cavero, M. (2005). Les mots du transport et de la gestion : approche sémantique-contextuelle. Meta, 50(4). https://doi.org/10.7202/019915ar
Résumé de l'article

Les exigences inhérentes au traitement automatique des langues ont mis en exergue la nécessité de formaliser l'articulation forme / sens et impliquent une procédure descriptive de la langue susceptible de rendre compte finement du rôle et du fonctionnement sémantique, dans les discours, de chaque unite lexicale, puisque nous avons affaire à des langues vivantes.

Privilégiant une approche sémantique du lexique dans la ligne de Benveniste et nous appuyant sur un corpus de documents préalablement sélectionné appartenant aux domaines spécialisés objet d'analyse (transport - gestion), nous étudierons les changements sémantiques qui militent en faveur de la créativité néologique ainsi que les difficultés que cela pose dès une perspective traductologique. Cela nous permettra de conclure la façon dont les nouveaux lexèmes s'inscrivent dans le système linguistique considéré ; en définitive, le fonctionnement interactif langue / discours.
Ce document est protégé par la loi sur le droit d'auteur. L'utilisation des services d’Érudit (y compris la reproduction) est assujettie à sa politique d'utilisation que vous pouvez consulter en ligne.

https://apropos.erudit.org/fr/usagers/politique-dutilisation/ 


\title{
Les mots du transport et de la gestion: approche sémantique-contextuelle
}

\author{
MERCEDES EURRUTIA CAVERO \\ Université d'Alicante, Alicante, Espagne \\ mercedes.eurrutia@ua.es
}

\begin{abstract}
RESUME
Les exigences inhérentes au traitement automatique des langues ont mis en exergue la nécessité de formaliser l'articulation forme / sens et impliquent une procédure descriptive de la langue susceptible de rendre compte finement du rôle et du fonctionnement sémantique, dans les discours, de chaque unité lexicale, puisque nous avons affaire à des langues vivantes.

Privilégiant une approche sémantique du lexique dans la ligne de Benveniste et nous appuyant sur un corpus de documents préalablement sélectionné appartenant aux domaines spécialisés objet d'analyse (transport - gestion), nous étudierons les changements sémantiques qui militent en faveur de la créativité néologique ainsi que les difficultés que cela pose dès une perspective traductologique. Cela nous permettra de conclure la façon dont les nouveaux lexèmes s'inscrivent dans le système linguistique considéré ; en définitive, le fonctionnement interactif langue / discours.
\end{abstract}

\begin{abstract}
Automatic treatment of languages states the need to formalize the form / sense articulation. So that, is necessary to know how the semantic of the lexical units works in the speech, its evolution and its sociocultural elements that delimit their sense, as we are in front of a living language.

Taking as bases a corpus of documents previously selected on the specific scopes object of study (transport - management), and from a semantic approach in Benveniste line, we will analyze the semantic changes which has been put under this terminology, its present interpretation and the difficulties that raises from the translation. All of it will allow us to conclude the way the new lexemes are registered in the analysed linguistic system ; really, the interactive operation language / speech.
\end{abstract}

\section{MOTS-CLES/KEYWORDS}

approche sémantique, changement sémantique, fonctionnement interactif langue / discours, créativité néologique

Le lexique évolue dans une interaction constante entre langue et discours. Son étude implique l'adoption d'une approche non seulement syntagmatique, mais encore paradigmatique afin d'éviter une atomisation artificielle. Dans ce sens, l'examen des procédures néologiques constitue un champ privilégié d'exploration du fonctionnement sémantique des unités lexicales.

Notre objectif est de mettre au jour les processus en jeu dans la constitution de nouveaux sens, insistant sur les changements sémantiques qui opèrent au sein de la terminologie analysée ainsi que sur les problèmes que cela pose du point de vue de la traduction. Pour ce faire, nous partirons de l'opposition traditionnelle matérialisée par la dichotomie néologie formelle $v s$ néologie sémantique (Dubois et al. 1994), réactualisée par J. Rey-Debove (1998 : 144) sous la forme de néologie de signe et néologie de sens.

Une approche sémantique du lexique, dans la ligne de Benveniste (1966a, 1974b), nous permettra de montrer que la néologie ne se manifeste pas nécessairement à travers la production d'un nouveau signifiant : elle peut provenir de glissements progressifs du sens reposant sur une sélection des sèmes précédemment actualisés et se manifester, éventuellement, à travers des conversions, par des variations statutaires ou catégorielles, des changements de classe grammaticale ou de classe sémantique, car le système de la langue n'est pas une simple abstraction linguistique. 
Bien que le lexique soit souvent considéré comme le lieu par excellence de l'hétérogénéité, on envisagera ici qu'il ne se constitue pas de manière anarchique uniquement au gré des variations du réel, des besoins de la société ou des modifications dans les savoirs, mais qu'il est le résultat de la mise en oeuvre de procédures linguistiques complexes identifiables et dénombrables permettant une interconnexion entre forme et sens.

Nous observerons les valeurs de certains mots à partir de leurs occurrences dans des discours spécialisés faisant partie d'un corpus préalablement déterminé. Par l'exploration des réseaux tissés au sein de la terminologie concernant le domaine des transports et de la gestion, nous examinerons la procédure d'inscription des nouveaux lexèmes dans le système linguistique étudié; en définitive, le fonctionnement interactif langue / discours.

La néologie sémantique, origine de la polysémie, correspond à un mouvement interne qui satisfait cette nécessité existentielle pour la langue de conjuguer changement et stabilisation, extension et économie (F. Cusin-Berche 1999: 10). Dès qu'une forme nouvelle est intégrée au lexique, son sens évolue et se délie de la «prescription morphologique » fondatrice, l'écart étant particulièrement sensible lorsque l'unité produite s'inscrit dans un processus dénominatif. C'est ainsi que les mots wagon, phares et train perdent leur sens initial dans les expressions accrocher le bon wagon (prendre place dans la bonne échappée, dans celle qui sera couronnée de succès), allumer les phares (suspicion à l'égard d'un coureur dont le regard trahit apparemment la prise de quelque produit dopant), assurer le train (la manière d'aller, l'allure, celle du cheval, d'une monture et par extension, d'un cycliste), imposer le train (se placer en tête du peloton), respectivement quand on les emploie dans le domaine du cyclisme (P. Fabre 2004).

Les phénomènes de restriction, d'élargissement et de déplacement du sens sont à la base de la création des néologismes sémantiques. Le verbe aller, qui signifiait « marcher, se promener », a pris le sens plus général de « se déplacer» (P. R. 1990 : 50). De son côté, le verbe arriver voulait dire « atteindre le rivage», son sens s'est élargi pour signifier « atteindre un lieu quelconque » $(\mathrm{P} . \mathrm{R}$. 1990 : 124). Les mots passent facilement du sens abstrait au sens concret, du sens moral au sens physique. Ils commencent par indiquer une action, puis ils s'appliquent soit au résultat de cette action, soit à la cause qui la produit, soit à l'objet par lequel elle s'accomplit. Le mot dessert indiquait l'action de desservir, "faire le service de en parlant d'une voie de communication ou d'un moyen de transport », mais aussi « débarrasser une table des plats, des couverts qui ont été servis » (P. R. 1990 : 519) plus tard ce mot désignait « l'action de desservir la table » et actuellement il est employé dans le sens de « dernier service d'un repas, comportant fromages, pâtisserie et fruits » ou « mets sucré, fruits, pâtisserie servis après le fromage » et par extension, «moment de la fin du repas » dans des phrases telles que Elle est arrivée au dessert. Un phénomène similaire est arrivé avec le mot avenue qui signifiait d'abord « arrivée », puis « la voie par laquelle on arrive » $(\mathrm{P}$. R. 1990 : 142).

Les emprunts internes constituent également une bonne preuve, citons parmi d'autres des termes empruntés par l'informatique à divers domaines (AFITEP 1996) tels que l'architecture (plateforme, architecture parallèle...), le langage juridique (protocole), commercial (débit), sportif (caddie), etc. On pourrait ajouter à ces exemples, certains termes du courrier postal qui ont été transférés vers le courrier électronique tels que : boîte aux lettres collective, paquet, poster...

L'infléchissement du sens de ces termes en contexte, opéré par la sélection de sèmes afférents et révélé par la modification de l'affectation référentielle, confirme l'existence, sous la pression de l'environnement lexical et culturel, d'une dynamique sémantique similaire à celle qui est en oeuvre sur le plan morphologique dans la mesure où il y a sélection d'éléments existants (sèmes connotatifs dans le cas de la néologie sémantique, morphes dans le cas de la néologie formelle) et réemploi partiel qui donne lieu à une nouvelle combinaison. Dans les phrases : Elle vient d'ouvrir le paquet qu'on lui a envoyé / Il a touché un paquet de billets / Je lui lâche son paquet (au sens figuré, critique sévère et méritée) / (...) La création d'étiquettes et de jaquettes, support de l'écriture par paquets pour utiliser le graveur comme un disque dur... le mot paquet prend des sens tout à fait différents. Seul le contexte peut servir à l'interpréter correctement.

Il semble, en effet, que la polysémie d'une unité lexicale se développe à partir des sèmes connotatifs, dont les propriétés extrinsèques sont susceptibles de rendre compte. Cependant si les 
emplois non dénominatifs exhument les traits permanents constitutifs du signifié, l'existence d'usages dénominatifs divers est également révélatrice. Ainsi, 1'unité lexicale « souris », initialement « nom d'un petit mammifère rongeur » (P. Robert et al. 1990:770) ${ }^{1}$ a servi également à désigner « une femme avec laquelle un homme entretient des relations éphémères », « une partie charnue située à l'extrémité du gigot » et « un boîtier connecté à un micro-ordinateur». Du fait de ses multiples usages dénominatifs, ce terme n'actualise plus de manière constante le trait animé mais se trouve caractérisé par les qualités de « petitesse et de rondeur ». Ce qui, lors d'une première approche, pouvait paraître secondaire (les sèmes connotatifs) est susceptible de correspondre, par la suite, au noyau sémique de l'unité, lequel est trop abstrait et minimaliste pour fournir une définition différentielle donc pertinente et opérationnelle du lexème.

Loin d'être un phénomène perturbateur, la polysémie apparaît comme une donnée essentielle, voire existentielle, de la langue en général et non seulement du lexique. Les mots peuvent avoir deux sortes de sens, le sens propre et le sens figuré. L'étude du sens figuré est traditionnellement réservé à la rhétorique. Le passage du sens propre au sens figuré s'obtient par divers mécanismes qui donnent lieu à différents types de figures, parmi lesquelles on peut citer les métaphores, les métonymies et les synecdoques. Nous passerons en revue ces différents types de changement sémantique insistant sur leur importance dès la perspective traductologique.

Le rapport entre le domaine de la gestion et celui des voyages est représenté par de nombreuses métaphores. Quand on voyage on regarde le paysage; en informatique on regarde le paysage de données, "représentation tridimensionnelle de données, habituellement représentée sous formes de listes, de tableaux ou de graphiques à deux dimensions » (M. Ginguay et A. Lauret 1990 : 286). Pour se faire on se penche à la fenêtre, métaphore qui désigne en Informatique la « partie rectangulaire de l'écran d'un ordinateur à l'intérieur de laquelle sont affichées les informations relatives à une activité déterminée » (Journal officiel du 16 mars 1999).

L'informaticien qui a créé la surface multiple permettant de voir plusieurs textes ou programmes a certainement cherché un nom à son invention. Il avait en tête le concept de celle-ci et il a dû l'analyser en ses différents aspects. De fait, une fenêtre nous offre la vue d'une partie du monde informatisé, une autre permet une perspective différente, etc. Il existe donc une analogie apparente à une fenêtre, qui, comme le disait Flaubert, «en province, remplace les théâtres et la promenade » (Madame Bovary, cit. par P. R. 1990 : 770). L'évolution de cet exemple met en évidence les diverses étapes de toute innovation sémantique : une première étape basée sur le principe d'association (contiguïté, similarité...). La deuxième phase consiste à transférer le signifiant de ce signe associé au concept pour qui on cherchait un mot. Si l'innovation lexicale réussit à convaincre un groupe de locuteurs déterminé (ex. d'autres informaticiens, les chef de l'entreprise, les usagers du programme), elle s'établit comme signification supplémentaire du mot en question et le changement sémantique est complet.

La métaphore du paysage dans le contexte de la représentation visuelle de données rappelle l'expression nuage de points qui exploite également une métaphore visuelle d'un élément géographique d'application fréquente dans le domaine de la météorologie (nuage de condensation, d'orage, de pluie, nuage lourd, moutonné, nacré). Un nuage de points est « un graphisme à deux dimensions sur lequel chaque information est représentée par un point. Il permet de visualiser la répartition et la diffusion des données représentées »(M. Ginguay et A. Lauret 1990 : 193). Les exemples de métaphores de l'environnement sont nombreux en Informatique : habitat, monde, parloir, salon, village, communauté virtuelle... Tous des termes appartenant au langage courant qui sont employés dans ce domaine spécifique dans un sens restrictif.

L'ordinateur est un objet inanimé qui, par définition, ne peut être mis en marche ou en mouvement que par une intervention humaine. Or, les verbes et les substantifs du champ lexical du mouvement ou du déplacement, lorsqu'on les applique à des machines ou des appareils statiques, leur confèrent un léger souffle de vie. Dans ce domaine sémantique, on trouve aussi bien des termes ayant trait aux transports en commun, par la route ou le fer, qu'aux déplacements automobiles, à bicyclette, à pied ou à cheval, sans oublier la vague terminologique liée à la navigation.

Les voies informatiques ne sont pas ferrées mais leur nombre et leur variété laissent deviner un réseau d’une densité comparable. On regroupe ces voies sous le terme générique de voies de 
communication. Il y a bien sûr une voie principale et des voies secondaires ou auxiliaires, des voies directes et des voies de déroutement, et si nécessaire des voies de secours, ainsi que des voies d'entrée et de sortie et, si le flux des informations est trop abondant, on le dérivera vers des voies de débordement. Comme les voyageurs, les données informatiques se déplacent en un va-et-vient incessant. D'ailleurs, des voies spécialisées sont réservées à chaque flux, une voie d'aller et une voie de retour. Mais n'entre pas qui veut: il faut montrer le contrôleur d'entrée-sortie qui vérifie si l'on remplit bien les conditions d'entrée à la façon d'un contrôleur d'autobus ou de train.

Bien sûr, l'ordinateur maitrise ces déplacements sur cet entrelacs de voies par un système d'aiguillages, de passerelles, de ponts et de raccourcis. Voilà deux termes métaphoriques qui font allusion à l'infrastructure routière, aussi fréquente en informatique : pont, autoroutes, passerelle, voie, canal, routeur, inforoute... Ce dernier mot-valise dont la création a été motivée par économie pour remplacer la forme développée autoroutes de l'information est, elle-même, un calque de l'américain information highway (en anglais, infohigways).

On n'a pas besoin de billet pour prendre un bus, un bus de commande ou un bus de données, le mot de passe suffit. Il n'y a ni chauffeur ni machiniste, mais un conducteur, en l'occurrence un conducteur de raccordement qui cherchera son chemin - nous l'appellerons chemin de données au milieu d'une circulation particulièrement dense, la circulation de l'information. Il y observera de nombreux arrêts, programmés ou automatiques. Certains d'entre eux sont conditionnels et, pour en bénéficier, on recommande de placer dans les programmes des signaux d'arrêts. Quand le trafic de données est fluide, les temps d'attente sont très courts. On les mesure d'ailleurs en nanosecondes, c'est-à-dire en milliardièmes de seconde. Il faut néanmoins se placer en file d'attente.

Sur les routes roulent les automobiles aussi présentes en informatique. On parle de moteurs, de moteur de recherche, entendant par là, « le logiciel de recherche de sites Web et d'adresses Internet par mots-clés ou par thèmes » (M. Ginguay et A. Lauret 1990 : 145). Les métaphores automobiles prêtent à croire que 1'ordinateur appartient à la famille des bolides de course. Il ne dédaigne pas les démarrages à chaud et, même s'il ne possède que des roues d'impression sur ses imprimantes d'un autre âge, il tourne à plusieurs vitesses de pointe, une vitesse maximale, une vitesse de transmission et une vitesse d'alimentation. Bien évidemment, pour évoluer à ces vitesses, ces prétendus bolides exigent des circuits. Il s’agira dans leur cas de circuits imprimés.

L'électronique parle quant à elle de circuits bouchon. Si l'on sait qu'un ordinateur souffre parfois d'être relié à des périphériques lents, on ne peut qu'être admiratif devant la similitude qu'évoquent ces mots avec nos propres boulevards périphériques. Pour des déplacements plus calmes, on peut emprunter de chemins de données, parfois aléatoires, qui vous conduiront peut-être, à la manière des chemins de traverse, vers de canaux de relâche où l'ordinateur pourra enfin se placer en position de repos.

L'Internet et les réseaux de télécommunications ont ajouté une nouvelle dimension à la notion de circulation, celle de la navigation maritime et aérienne.

La métaphore de la navigation est présente partout : pilote électronique, navigateur, naviguer, surfer sur Internet, pirate informatique... Au début du $\mathrm{XX}^{\mathrm{e}}$ siècle, l'aéronautique naissante a adopté une bonne part de la terminologie maritime : pilote, navigateur, steward pour désigner le personnel navigant, gouvernail, hélice, voilure pour certaines parties de l'avion et des tournures comme monter à bord, embarquer, flotte aérienne. Rien d'étonnant puisque l'on parlait alors de navigation aérienne par opposition à la navigation maritime classique. C'est ainsi que le terme pilote " celui qui dirige un navire » désigne par analogie, « la personne qui conduit un avion ou un aéronef » $(\mathrm{P}$. $\mathrm{R}$. 1990 : 1437). Ce terme doit être interprété en informatique comme le «programme qui permet à un périphérique de fonctionner en association à un ordinateur» (M. Ginguay et A. Lauret 1990 : 295). Il s'agit d'un calque de l'anglais driver. Dans son sens propre, driver désigne le conducteur d'un véhicule automobile ou d'un train. Le calque français pilote fait glisser la métaphore vers le domaine maritime et aéronautique. Déjà en usage depuis les années 80 , il s'agit d'un terme précurseur de la notion de navigation sur réseau. Il peut également être rapproché de la notion de pilote automatique qui désigne un équipement capable d'assurer le fonctionnement d'un navire ou avion sans intervention humaine. 
Plus tard, les premiers conquérants de l'espace ont été des pilotes d'essai. Il ne faut donc pas être surpris qu'on ait choisi pour désigner un terme suffixé en -naute (astronaute, cosmonaute, spationaute) du grec nautès, marin, navigateur.

Aujourd'hui, l'espace à conquérir est un espace cybernétique, le cyberespace. Ses conquérants sont des cybernautes (cybernétique + astronaute) ou internautes (Internet + astronaute). D’ailleurs, le terme cybernétique a également pour racine un terme de marine, le gouvernail de navire. On comprend donc très bien que des expressions comme naviguer sur Internet ou logiciel de navigation se soient aisément implantées en français.

En ce qui concerne le verbe surfer employé dans le même registre, il ajoute une dimension sportive à l'activité de la navigation. Aux États-Unis, parallèlement à l'expression surfing the Internet sévit une expression peut-être moins compétitive et plus décontractée, crusing the Internet qui signifie littéralement « croiser, faire une croisière sur Internet» (G. Colpron 1982 : 132). Elle exprime mieux les aspects promenade et découverte d'un parcours sur le Web. À l'image du pilote de vaisseau aérien qui a besoin d'un bon navigateur pour arriver à bon port, le cybernaute dispose également d'instruments de navigation appelés logiciel de navigation ou navigateur. J. Colombain écrit un livre intitulé Surfer sur Internet et parle de cette « drôle d'expression » dans les termes suivants : «Surfer sur Internet, désigne la manière originale dont on « navigue » sur le réseau informatique mondial : comme un surfeur sur la crête des vagues, l'internaute se déplace de site en site en cliquant avec sa souris » (J. Colombain 1999: 3).

On évoque la navigation routière en tant qu'ensemble de systèmes et de technologies conçus pour faciliter les déplacements automobiles et on parle de navigation automobile, de système d'aide à la navigation automobile, de navigateur routier... mais aussi de cartographie numérisée, terme qui sert à désigner la réalisation de cartes numérisées ou numériques à partir de cartes et plans sur support papier, de pilote électronique, de guide de navigation, de navion, etc.

Les voies de navigation se voient parfois soumises à l'assaut des pirates « aventurier qui courait les mers pour piller les navires » (P. R. $1990: 1442)$; en informatique, « spécialiste qui cherche à s'introduire dans des réseaux protégés en perçant les mots de passe et autres barrières de sécurité dans des intentions ludiques ou malveillantes » (M. Ginguay et A. Lauret $1990:$ 300) : «Comme par le passé, le piratage peut être un simple canular ou un acte de vandalisme très destructeur » $(L e$ Monde, 25-26 août, 1996). Et les spécialistes distinguent même plusieurs catégories de pirates informatiques : le hacker, le cracker et le phreaker (termes empruntés à l'anglais).

Pour se protéger et pouvoir jouir d'une belle croisière (sur Internet), il faut engager un organisateur compétent ; en informatique, « l'ordinateur de poche de la taille d'une grosse calculette qui comprend généralement des fonctions d'agenda électronique, de calculatrice, de répertoire d'adresses et de numéros de téléphones personnels, d'horloge et de signal de rendezvous » (M. Ginguay et A. Lauret 1990 : 214).

Tout à fait lié au domaine du transport, on trouve celui de l'hôtellerie et de la restauration, auquel on a toujours recours lors d'un voyage (hôte, client, serveur, client-serveur...). En informatique, le terme composé client-serveur a été adopté par analogie avec le scénario où le client est servi par le serveur pour désigner l'architecture d'un réseau informatique qui comprend un ou des ordinateur(s) serveur(s) et un grand nombre d'ordinateurs clients qui communiquent entre eux à l'aide de logiciels d'interrogation (les ordinateurs clients sont des terminaux standards, alors que l'ordinateur serveur est caractérisé par sa puissance de stokage). À côté de cette métaphore, celle de la gastronomie ne pouvait pas manquer (cookie, menu, grain de café...) : on parle de cookie " petit programme informatique » (en plus des bogues, les internautes risquent d'être victimes des cookies); en anglais petit gâteau sec. Quant à l'unité lexicale grain de café, calque de Java Beain, elle doit être interprétée en informatique en tant que « composant portable écrit en langage Java et intégré à une application qui permet d'interagir avec un composant du même type logé dans une autre application » (M. Ginguay et A. Lauret 1990 : 98). Il faut chercher cet emploi dans les sens argotiques de ce mot (java pour café - caoua).

Les exemples jusqu'ici analysés montrent la force et la faiblesse de la motivation métaphorique : d'un côté, il y a la valeur picturale, le pouvoir d'évocation, le caractère concret et vif ; de l'autre côté, on constate le manque de systématisation, l'absence d'indications objectives et, en particulier, l'ambiguiité, c'est-à-dire l'applicabilité parfois d'une même métaphore à une quantité trop 
élevée de cas différents. C'est le cas de oeil-de-boeuf qui peut désigner une fenêtre ronde (ex. d'un bateau) mais aussi une plante, un animal et une pierre.

A force d'être répétées, les métaphores peuvent s'user : le sens métaphorique devient banal et il entre dans le vocabulaire. Dans ces cas on parle de métaphores lexicalisées, c'est-à-dire celles que donnent les dictionnaires comme faisant partie des significations du mot.

Aux métaphores lexicalisées s'en ajoutent d'autres que les sujets parlants produisent librement. Quand, lors d'un voyage, on arrête la voiture pendant quelques minutes pour se reposer et qu'on s'allonge dans un pré, le locuteur peut, provisoirement, le dénommer lit. Dans ce cas, il s'agit d'une métaphore occasionnelle qui ne peut se comprendre que dans ce contexte particulier.

La productivité de la métaphore dans le processus de création des néologismes sémantiques ne cesse de nous surprendre. Fondée sur la ressemblance et la similitude, la métaphore s'appuie sur des analogies existantes dans le réel ou construites par le sujet parlant : on parle d'un moteur en $V$ (par motivation graphique), de passage clouté, "passage pour piétons » (P. R. $1990: 328$ ) autrefois limité par des grosses têtes de clous actuellement remplacés par des lignes blanches ou de feu, d'abord flamme d'un feu ou dégagement d'énergie calorifique et de lumière (P. R. 1990 : 775) et, par extension, toute source de lumière, signal lumineux (feu vert de tribord, feu rouge de bâbord), phare (les feux d'un avion, d'une voiture, feu de position, de stationnement, feux clignotants) ou instruments servant à régler la circulation routière (feu rouge, passage interdit ; feu jaune, ralentir ; feu vert, voie libre). Il est aussi intéressant d'examiner à ce propos le mot aile (métaphore zoomorphique / forme-fonction). Au sens propre, ailes désigne « chacun des organes du vol chez les oiseaux, les chauves-souris, les insectes » (P. R. 1990 : 41). Parmi les sens seconds de ce mot, on trouve entre autres « chacun des plans de sustentation d'un avion » (ibidem); on parle alors des ailes d'avion (nos places se trouvent à côté de l'aile). Malgré les différences qui distinguent ces deux sortes d'ailes (battantes et fixes), on peut trouver des ressemblances entre elles à la fois au niveau de leur forme et au niveau de leur fonction. Le mot est employé aussi pour d'autres référents, sur la base d'une simple ressemblance d'aspect : ailes du nez, ailes d'une armée ou d'une équipe sportive, ailes d'un bâtiment, ailes d'un moulin de vent, aile à réaction, aile de dérive, aile porte-à-faux, ailes d'hélice, etc. C'est donc par métaphore que dans le mot aile tous ces sens se trouvent reliés. Tous ces signifiés ont des sèmes en commun qui s’actualisent de façon différente selon le contexte.

Les métaphores animalières ou zoomorphiques montrent l'immixtion du vivant dans le monde informatique : mouchard électronique, souris (selon Douglas Engelbart, son créateur, il aurait reconnu dans le fil émergeant de ce boîtier la queue de ce petit rongeur commun), puce (vocable familier désignant la pastille de silicium qui contient un ensemble de circuits intégrés, a été choisi par analogie avec l'insecte sauteur bien connu pour deux raisons, l'analogie de couleur brune et la présence de griffes de fixation placées sous la pastille rappelant étrangement les pattes de la puce. Cette référence est bien française car le mot d'origine, chip, qui, au sens propre signifie fragment, copeau, lamelle, éclat, n'a rien à avoir avec le règne du vivant), virus (série d'instructions destinée à perturber ou détruire le bon fonctionnement d'un ordinateur de la même manière qu'un virus provoque une infection). Quant à la notion toile d'araignée (Web) a entraîné dans son sillage d'utilisation d'une nouvelle métaphore animale, l'araignée, pour désigner un moteur de recherche qui parcourt le Web et l'indexe. L'araignée, à la différence de la punaise et des virus, est utile et positive dans l'environnement informatique : une araignée est un moteur de recherche d'un type particulier qui agit automatiquement une fois lancé.

Mais le monde du vivant est surtout représenté par les nombreuses métaphores antropomorphiques : intelligence, mémoire, comprendre, lire, assistant, nez électronique, costume de données... L'intelligence est, par définition, une faculté réservée aux êtres vivants mais depuis quelques années, l'adjectif intelligent est de plus en plus accolé aux objets les plus divers (terminaux intelligents, bureaux intelligents, matériaux intelligents...). On pourrait situer l'introduction de la notion d'intelligence artificielle en 1960 et on la doit à Jacques Pitrat. L'informatique pullule de termes liés à des activités humaines nécessitant une forme d'intelligence. Les ordinateurs calculent, lisent, copient, classent, comparent... À tel point qu'aujourd 'hui on peut qualifier d'intelligent tout dispositif qui possède des moyens autonomes de traitement de l’information ; d’où le grand nombre de termes métaphoriques ainsi créés. 
On observe l'importance des changements sémantiques en tout ce qui concerne le secteur administratif et gestionnaire. Des mots tels que : dossier, bureau, fichier, document, gomme, ciseaux, presse-papiers, papier peint, corbeille, poste de travail, télétravail, professionnel itinérant, crayon-lecteur... en offrent un bon exemple. Prenons le mot bureau « table sur laquelle on écrit »- « pièce où est installée la table de travail » (P. R. 1990 : 226). Il s'agit d'un dérivé formé à partir du mot bure par adjonction du suffixe-eau. Bureau, qui actuellement possède plusieurs significations, désignait autrefois une étoffe de laine foncée et grossière. Son origine remonte au XIII ${ }^{\mathrm{e}}$ siècle (A. Dauzat et al. 1993 : 157), époque pré-gutenbergienne où la diffusion du livre était l'oeuvre de copistes, généralement des moines. Ces moines déposaient sur leur table de travail un carré d'étoffe de bure, la même bure qui servait à confectionner leur habit. Les gens pauvres avaient l'habitude de porter des vêtements de bureau. Cette grosse étoffe de laine servait aussi à faire des tapis de table et particulièrement ceux des tables auxquelles on s'asseyait pour délibérer, pour examiner une question, pour juger. Par métonymie (partie - tout), du tapis qui couvrait la table, le nom est passé à la table elle-même, puis à la pièce où se trouve cette table, puis aux personnes qui travaillent dans cette pièce et enfin au service administratif. Tous ces sens, qui apparemment ont été donnés au mot les uns après les autres, subsistent aujourd’hui, alors que le sens initial, dit sens premier, a été éliminé il y a longtemps. Historiquement, le sens de bureau a évolué au fil des siècles par extensions progressives, l'extension sémantique se faisant parallèlement à une extension physique de l'objet. L'ordinateur a permis de poursuivre l'extension sémantique mais a rompu avec le processus d'extension physique. En informatique, et, en particulier dans l'environnement Windows, on appelle bureau «l'espace de travail que constitue l'écran de l'ordinateur au moment de l'ouverture d'une session » (M. Ginguay et A. Lauret 1990 : 62). La métaphore du bureau (élément de mobilier et local) est renforcée par l'utilisation dans le même contexte des métaphores: dossier, fichier... Pour démarrer une session de travail, le « scribe » moderne ouvre son bureau un peu comme le moine copiste étalait son carré de bure sur sa table de bois. Cette première extension sémantique correspond donc à une réduction physique de la notion de bureau (en particulier dans le sens d'édifice). Il en existe une seconde qui correspond à un éclatement de l'espace matériel du bureau. C'est celle de bureau mobile. Le bureau mobile est avant tout un bureau, avec ses équipements habituels, logé dans un véhicule automobile spécialement aménagé. Mais c'est aussi une approche nouvelle du concept de travail de bureau qui s'effectue à l'extérieur de l'immeuble de bureau traditionnel. Les notions de télétravail, de bureautique sans fil, de professionnel itinérant et d'objet nomade en font partie.

Les métaphores impliquent tel qu'on peut constater par des exemples ci-dessus cités un degré de liberté qui n'existe pas dans les métonymies (un Pentium -un ordinateur Pentium-, un portable un ordinateur portable ou un téléphone portable-, tous les deux créés par absortion lexicale ou contiguiité syntagmatique) ou les synecdoques. La tendance à la réduction des groupes fréquemment répétés entraîne des phénomènes de changement sémantique qui permettent de faire passer le message à moindres frais. Elle se manifeste en particulier dans la métonymie. Métro désigne aujourd 'hui « tout chemin de fer urbain, généralement souterrain » (P. R. 1990: 1193). Ce n'est que l'abréviation de métropolitain, dont la signification était « de la capitale ». Cet adjectif, par ellipse du nom composé qu'il déterminait, s'est chargé du sens de l'expression entière : chemin de fer métropolitain, trop longue pour être aisément maniable, elle a été une première fois abrégée au profit de l'adjectif, puis, pour la même raison, ce dernier l'a été à son tour, quitte à perdre ainsi toute motivation : c'est une forme devenue arbitraire.

Jouant sur les relations de contiguité entre objets, individus ou événements, la synecdoque remplace le nom de l'un des deux objets par celui de l'autre (M.- F. Mortureux 1997 : 118). Les deux objets, à la différence de ce qui se passe dans la métonymie, ne sont pas indépendant 1'un de l'autre mais liés par un lien de type définitionnel. Un exemple de synecdoque illustrera cette notion (la partie pour le tout) : voile pour bateau. Dans la phrase « un vaisseau comprend une coque et des voiles », le terme voile est parfaitement compréhensible. Par contre, quand on dit « il voit cinquante voiles (vaisseaux) à l'horizon », le sens figuré ou sens second ne peut se comprendre que dans un contexte particulier. Il faut toutefois prendre garde à ne pas conclure que le sens non figuré soit une expression première considéré comme «normale », ce qui pourrait laisser penser qu' un langage idéal serait dépourvu de figures. La figure ne doit donc pas être définie comme un écart par rapport 
au bon usage. Bien que beaucoup plus rarement que la métonymie et la métaphore, les synecdoques provoquent parfois (tel qu'on l'observe par des exemples), des changements de sens. C'est aussi le cas du mot nef qui désignait originellement un bateau. Ce mot s'est spécialisé pour désigner un type particulier de bateau à grandes voiles. Le mot nef, au lieu de s'appliquer au genre, ne s’applique donc plus qu'à une espèce.

Finalement on fera allusion au transfert co-hyponimique. On parle de ce changement sémantique lorsqu'on transmet le nom d'un concept à un concept qui se trouve au même niveau d'abstraction dans une taxinomie populaire à cause d'une similarité assez marquée entre les référents. C'est un type de changement sémantique assez fréquent au niveau du discours mais qui ne va que rarement jusqu'à la lexicalisation. Il se produit souvent quand un groupe de locuteurs superpose sa nomenclature habituelle à un milieu géographique différent mais présentant des référents similaires : au Québec, sur les panneaux de signalisation routière, on trouve systématiquement, à la place du mot anglais stop, le mot arrêt ; de même faire du pouce, expression dans laquelle il faut reconnaître le verbe anglais to thum, est employée dans le sens de faire de l'auto-stop. On dit qu'on part en pouce ce qui se dirait en Afrique, voyager par occasion ou qu'on trouve une pouce, c'est-à-dire, une auto qui vous prend à son bord. Dans tous les cas on est un pouceur, non un auto-stoppeur.

Quand on se déplace en France, « on change de place » tandis qu'au Tchad « on déménage »; en France « on fait le plein » à une station-service, au Sénégal, à une essencerie.

Tous ces exemples font preuve de la capacité de l'esprit humain de découvrir sans cesse des analogies qui permettent d'utiliser un mot existant avec un sens nouveau, de l'appliquer à de nouveaux référents. La polysémie semble donc résulter d'une accumulation de sens apparus successivement. Puisqu'il est presque impossible de créer autant de mots nouveaux qu'il y a de référents nouveaux, les sujets parlants augmentent considérablement, à l'aide de la polysémisation, les possibilités des mots déjà existants. Une langue totalement monosémique serait impensable, car elle posséderait un lexique pratiquement infini. Le lexique évolue. Parfois les mots voient leur sens se diversifier ou se simplifier ; certains mots sont oubliés et finissent par disparaître, remplacés ou non par d'autres. Il faut considérer que, quand un sens d'un mot polysémique s'élimine, ce n'est pas forcément le sens premier ou le sens apparemment le plus ancien. L'ensemble des appréciations véhiculées sur un objet donné sont des indices importants pour accéder à la représentation que l'on a de cet objet et sont constitutives d'une valeur ajoutée au sens du mot qui le dénommé (L. Bonicel et al. 2003 : 131). On accède donc au sens d'une unité lexicale grâce à ses actualisations dans des contextes divers, à l'étude de sa distribution dans la phrase. Mais il faut sans doute considérer qu'une unité lexicale ne se prête pas à n'importe quelle actualisation. À notre avis, ce ne sont pas les emplois qui déterminent le(s) sens, mais le(s) sens qui conditionne(nt) les emplois. Les mots en discours sont susceptibles de variations sémantiques selon le contexte ; réciproquement l'interprétation d'un énoncé s'appuie sur l’identité lexicale de ses composants, identité parfois corrélative à des statuts grammaticaux.

Par rapport aux rôles du cotexte et du contexte dans la résolution d'une ambiguité lexicale ayant pour origine l'existence d'unités homonymiques, on constate avec M. Charolles (1996 : 162) qu'une phrase telle que « À l'aéroport de Strasbourg, il y a dix vols par jour » contient des indices cotextuels qui invitent à saisir vol en relation avec l'aviation, mais pourrait être comprise comme véhiculant des informations sur les vols commis par des voleurs, cela pour des raisons liées à une fréquence d'emploi plus élevée entraînant « une plus grande saillance dans le lexique mental » (ibidem). Cependant, eu égard à notre préoccupation de délimiter l'influence du cotexte par rapport à celle du contexte, nous n'en concluons pas que l'interprétation du lexème vol est indépendante du contexte, mais qu'elle est imperméable à la suggestion cotextuelle, parce que dominée ou conditionnée par la contextualisation mentale. Aussi constate-t-on que le cotexte minimaliste ne permet pas toujours de résoudre l'ambiguité lexicale, de réduire la polysémie initiale et donc de choisir le sens idoine lorsqu'un signifiant est susceptible d'être associé à deux signifiés perçus dans la conscience synchronique comme étrangers l'un à l'autre.

Quant aux désignations métaphoriques qui, par définition, induisent une ambivalence de l'unité lexicale concernée, elles imposent un transfert référentiel étroitement associé au maintien, au moins partiel, du sens antérieur : «Les voisins d’à côté font trop de bruit, vous venez de perdre 
votre carte bleue, vous comptez démissionner de votre emploi et monter une société... bonjour la paperasse à caractère juridique! Heureusement, EBP a conçu ses assistants juridiques pour vous » (SVM, février 1995). Le mot assistant désigne en informatique, tel qu'on l'observe dans cet exemple, le logiciel ou utilitaire informatique qui permet de réaliser plus facilement une tâche donnée. On note que, dans ce cas, le rétablissement du contexte d'énonciation ne contredit pas les données cotextuelles; il permet de sélectionner les sèmes à actualiser. Toutefois, la lexicalisation de la métaphore, institutionnalisant la polysémie du lexème, va faire émerger des cotextes spécifiques témoignant de la constitution d'une nouvelle acception tels que « Je vous présente l'assitant juridique qui va me représenter lors du jugement » (dans une conversation) qui sont susceptibles de signaler le contexte particulier de validité et d'actualiser les sèmes contenus potentiellement dans assistant juridique. Tout changement référentiel exerce une influence sur l'appréhension du sémantisme de l'unité lexicale concernée par cette transposition et devient susceptible de favoriser la mise au jour des sèmes spécifiques occultés précédemment.

Pourtant, un rapide parcours permet de vérifier que lorsque les données situationnelles entrent en contradiction avec les données linguistiques, il n'en résulte pas de modification du sens des unités lexicales mais un changement d'ordre pragmatique pesant sur l'interprétation de l'énoncé et qu'en revanche, une unité lexicale faisant l'objet d'un nouvel emploi, c'est-à-dire utilisée dans un contexte jusqu'alors inhabituel, peut, tout en conservant le même environnement linguistique et le même noyau sémantique, acquérir des sèmes complémentaires qui infléchissent légèrement le contenu du sémème, soit par le biais de la métaphore (fenêtre), soit par le biais de la métonymie (portable)... Nous avons également remarqué que lorsqu'un signifiant dispose en langue de deux signifiés (vol), l'environnement linguistique du mot observé peut militer en faveur d'un des sens, lequel est susceptible d’être invalidé sous l'influence du contexte énonciatif ou cognitif.

En guise de conclusion, on pourrait dire que l'examen des procédures néologiques que nous avons mené met en exergue une relative systématicité dans le développement du lexique sur le plan sémantique. Il tend également à convaincre d'une continuité en faveur de l'existence d'une identité lexicale conçue comme un signifié construit en et par la langue, susceptible d'évoluer en discours mais dont le sens est conditionné pour partie par le système. La dimension grammaticale (particulièrement sollicitée à travers les phénomènes de conversion, de lexicalisation et de grammaticalisation) permet d'identifier certains mouvements lexicaux internes non repérables morphologiquement. On remarque à ce propos que la grammaticalisation est traditionnellement appréhendée comme un appauvrissement de sens, alors que la lexicalisation associée à la « dégrammaticalisation » est pressentie comme un enrichissement.

Actuellement, on ne dispose pas de définition vraiment satisfaisante des sens grammaticaux ni des sens syntaxiques ; c'est pourquoi on ne peut corréler systématiquement catégorie grammaticale, position syntaxique et sens lexical. Néanmoins, dans une perspective exploratoire d'ordre lexicologique, les approches grammaticale et syntaxique constituent des outils, imparfaits mais indispensables, pour mettre au jour des indices permettant d'appréhender le(s) sens d'un lexème à partir de ses actualisations discursives.

\section{NOTES}

1. Par la suite on fera référence à cet ouvrage par l'abréviation P.R.

\section{REFERENCES}

AFITEP (1996) : Dictionnaire de management de projet, $3^{\mathrm{e}}$ éd., Paris, AFNOR. Aguadero, F. (1998) : Diccionario de términos multimedia, Madrid, Acento. Aguado Cea, G. (1993) : Diccionario comentado de terminología informática, Madrid, Paraninfo. Alarcón ÁlvareZ, E. (2004) : Diccionario de términos informáticos e Internet, Madrid, Anaya. Benveniste, É. (1966) : Problèmes de linguistique générale, tome I, Paris, Gallimard. 
Benveniste, É. (1974) : Problèmes de linguistique générale, tome II, Paris, Gallimard.

Blank, A. (1999) : «Les principes d'association et la structure du lexique », Studi di Linguistica Teorica e Applicata XXVIII, p. 199-223.

BONICEL, L. et al. (2003) : Mots et lexiculture, Paris, Honoré Champion Éditeur.

Colombain, J. (1999) : Surfer sur Internet, coll. « les essentiels », Toulouse, Milan.

COLPRON, G. (1982): Dictionnaire des anglicismes, Québec, Beauchemin.

CUSIN-BERCHE, F. (1998) : Le management par les mots. Approche sociolinguistique de la néologie, Paris,

L'Harmattan.

CUSIN-BERCHE, F. (1999) : «Le lexique en mouvement: création lexicale et production sémantique », Langages

136, p. 5-26, Paris, Larousse.

Charles, R., S. Steelandt (1994) : Le vocabulaire français, Paris, Nathan.

Charolles, M. (1996) : «Quand intervient le contexte dans la résolution des ambiguïtés? », Scolia 6, p. 163-184.

DAuZAT, A. et al. (1964, 1993) : Dictionnaire étymologique et historique du français, Paris, Larousse.

DEPCKER, L. (1999) : Guide des mots francophones, coll. «Point Virgule », Paris, Éditions du Seuil.

Detrie, C. (2001) : Du sens dans le processus métaphorique, Paris, Honoré Champion Éditeur.

DuBoIs, J. et al. (1994) : Dictionnaire de linguistique et des sciences du langage, Paris, Larousse.

FABRE, P. (2004) : Expressions du cyclisme, Paris, Bonneton.

Guinguay, M. et A. Lauret (1990) : Dictionnaire d'informatique, 4e éd., Paris, Masson.

(1999) : Journal officiel, (16 mars).

LAKOFF, G. et M. JOHNSON (1985) : Les métaphores dans la vie quotidienne, Paris, Minuit.

(1996) : Le Monde, (25-26 août).

MortureuX, M.-F. (1997) : La lexicologie entre langue et discours, coll. « Campus », Paris, SEDES.

NyCKeES, V. (1998) : La sémantique, Paris, Belin.

Polguere, A. (2003) : Lexicologie et sémantique lexicale, coll. « paramètres », Montréal, Les Presses de

1'Université de Montréal.

PotTiER, B. (1992) : Sémantique générale, Paris, PUF.

Reboul, S. (1991) : Le Vocabulaire de la télématique, du discours au lexique, Paris, Larousse.

ReY-Debove, J. (1998) : La linguistique du signe, Paris, Armand Colin.

Robert, P. et al. (1989, 1990) : Le Petit Robert 1, 2e éd., Paris, Montréal, Le Robert.

SABlayrolles, J.-F. (2000) : La Néologie en français contemporain, Paris, Honoré Champion Éditeur.

SABlayrolles, J.-F. et al. (2003) : L'innovation lexicale, Paris, Honoré Champion Éditeur.

(1995) : SVM, (février).

TEJERA, P. (1994) : Diccionario enciclopédico de informática, México, Editorial Iberoamericana. 